ORIGINAL ARTICLE

\title{
LYVE-1 immunohistochemical assessment of lymphangiogenesis in endometrial and lung cancer
}

M I Koukourakis, A Giatromanolaki, E Sivridis, C Simopoulos, K C Gatter, A L Harris, D G Jackson

See end of article for authors' affiliations

.....................

Correspondence to: Dr M I Koukourakis, Tumour and Angiogenesis Research Group, PO Box 12, Alexandroupolis 68100, Greece; targ@her. forthnet.gr

Accepted for publication 15 June 2004

\begin{abstract}
Aims/Methods: Normal and malignant pulmonary and endometrial tissues were analysed for lymphatic vessels to assess the process of lymphangiogenesis and its role at these sites, using specific immunostaining for LYVE-1 and the panendothelial marker CD31.

Results: Lymphatics were clearly demonstrated in some normal tissues (myometrium, bronchial submucosa, and intestinal submucosa), but not in others (endometrium and alveolar tissue). LYVE-1 positive lymphatic vessels were detected at the tumour periphery of endometrial and lung carcinomas, but not within the main tumour mass. Double staining for LYVE-1 and the MIB1 proliferation marker revealed a higher proliferation index in lymphatic endothelial cells at the invading front of endometrial carcinomas, compared with myometrial areas distal to the tumour. Lung and endometrial carcinomas did not have an intratumorous lymphatic network.

Conclusions: Although lymphangiogenesis may occur at the invading tumour front, incorporated lymphatics do not survive. Therefore, the dissemination of cancer cells through the lymphatics may occur by invasion of peripheral cancer cells into the adjacent normal lymphatics, or through shunts eventually produced at the invading tumour front as a consequence of active angiogenesis and lymphangiogenesis.
\end{abstract}

$\mathrm{S}$ pecific monoclonal antibodies (MoAbs) recognising the factor VIII related antigen, CD31, and CD34 endothelial cell membrane antigens have been used to assess intratumorous micovessel density, a direct marker of tumour angiogenic activity. ${ }^{1}$ However, these MoAbs do not discriminate between the vascular and the lymphatic component of the intratumorous vasculature, although CD31 has a lower affinity for lymphatics. ${ }^{2}$ Lymphatic spread of the disease is assumed to occur through cancer cell permeation of intratumorous lymphatics, thus reaching the regional lymph nodes. However, it is unclear whether lymphatic dissemination is dependent upon cancer cell infiltration of preexisting lymphatic vessels or newly formed ones, originating from those of the normal surrounding tissues.

"A selective marker of the lymphatic endothelium, LYVE-1, has recently been identified"

This last process, "lymphangiogenesis", has been difficult to investigate up to now because there was a lack of specific MoAbs selectively recognising the lymphatic endothelium. Recently, the vascular endothelial growth factor (VEGF) receptor 3 (VEGFR-3) was recognised as a specific lymphatic marker binding to VEGF-C. ${ }^{3}$ However, although VEGFR-3 is not expressed in the non-lymphatic vasculature in postnatal life, it is commonly expressed in the embryonic vasculature. ${ }^{4}$ Activation of the intratumorous vasculature allows reexpression of several suppressed genes and reduces the specificity of VEGFR-3 as a marker for tumour lymphatics. ${ }^{56}$ Indeed, in a recent study, VEGFR-3 was extensively distributed in benign and malignant vascular tumours of both blood and lymphatic origin, and also in the tumour vasculature. $^{7}$

A selective marker of the lymphatic endothelium, LYVE-1, has recently been identified. ${ }^{8}$ LYVE-1 is a surface endocytic receptor for hyaluronan, ${ }^{9}$ which shares $41 \%$ homology with the metastasis related CD44 molecule. Hyaluronan is an extracellular glycosaminoglycan involved in cell adhesion and migration. ${ }^{8}$ The highly selective expression of LYVE-1 in the lymphatics is probably attributable to the important role of the lymphatic system in the metabolism of hyaluronan. ${ }^{10}{ }^{11}$ More than $80 \%$ of tissue hyaluronan is degraded within the lymph nodes. LYVE-1 is probably a key receptor responsible for the uptake and transport of hyaluronan in the lymph.

It is not known whether there are differences in the angiogenic and lymphangiogenic activity of different tumours..$^{12}$ Certainly, the existence of lymphangiogenesis could explain the tendency of several tumours to spread preferentially to the lymph nodes rather than metastasising to distant organs. Recent experimental studies suggest that lymphangiogenesis does occur in experimental tumours and transfection of VEGF-C into tumour cell lines increases lymphatic density and lymphatic invasion, tumour growth, and metastasis..$^{14}$ In our present study, we investigated lymphangiogenesis in human lung and endometrial cancer, using immunohistochemistry with an antibody directed against LYVE-1. These tumours were chosen because we had extensively studied their vasculature in previous studies.

\section{MATERIALS AND METHODS}

Formalin fixed, paraffin wax embedded tissues from 28 endometrial adenocarcinomas of the endometrioid cell type were retrieved from the archives of the department of pathology, Democritus University of Thrace, Alexandroupolis, Greece. All cases in the series were stage I disease so that lymphangiogenesis could be assessed in the early stages of endometrial tumour development. The patients were treated with total abdominal hysterectomy without lymph node dissection.

Similarly, surgical material from operable (T1,2-N0, stage 1) non-small cell lung carcinomas (19 adenocarcinomas and

Abbreviations: APAAP, alkaline phosphatase/antialkaline phosphatase; DAB, diaminobenzidine; MoAb, monoclonal antibody; PBS, phosphate buffered saline; TBS, Tris buffered saline; VEGF, vascular endothelial growth factor; VEGFR, vascular endothelial growth factor receptor 
49 squamous cell carcinomas) was also retrieved from the department of cellular science, University of Oxford, UK. In addition, 15 surgical samples from normal endometrium of various phases of the menstrual cycle and eight samples from normal lungs (from patients who underwent surgery for reasons other than cancer) were retrieved.

\section{LYVE-1 immunohistochemistry}

Mouse antihuman LYVE-1 clone 8C (IgGl isotype) was raised against an immunoglobulin fusion protein of human LYVE-1 lymph vessel endothelial hyaluronan receptor. ${ }^{8}$ Sections were dewaxed and antigen retrieval was carried out by microwaving in Dako retrieval buffer ( $\mathrm{pH}$ 6.0; Dako, Glostrup, Denmark), three times for four minutes each. Slides were incubated in phosphate buffered saline (PBS) with 5\% human serum for five minutes. Peroxidase was quenched with methanol and $3 \% \mathrm{H}_{2} \mathrm{O}_{2}$ for 15 minutes. The primary antibody (in PBS plus 5\% fetal calf serum) was applied for 45 minutes. After washing with PBS, sections were incubated with a secondary antimouse horseradish peroxidase conjugated antibody (Kwik Biotinylated Secondary; ShandonUpshaw, Pittsburgh, Pennsylvania, USA) for 15 minutes and washed in PBS. The colour was developed by a 15 minute incubation with diaminobenzidine (DAB) solution and sections were weakly counterstained with haematoxylin. Normal tissue sections from the small intestine were used as positive controls. Normal mouse IgG was substituted for primary antibody as the negative control (same concentration as the test antibody).

\section{CD31 immunostaining}

The JC70 MoAb (Dako), recognising the CD31 panendothelial antigen (platelet/endothelial cell adhesion molecule) was used for microvessel and single endothelial cell staining on $5 \mu \mathrm{m}$ thick paraffin wax embedded sections. We used the alkaline phosphatase/antialkaline phosphatase (APAAP) procedure. Sections were dewaxed, rehydrated, and predigested with protease type XXIV for 20 minutes at $37^{\circ} \mathrm{C}$. JC70 ( $1 / 50$ dilution) was applied at room temperature for 30 minutes and washed in Tris buffered saline (TBS). Rabbit antimouse antibody at a dilution of $1 / 50(\mathrm{vol} / \mathrm{vol})$ was applied for 30 minutes, followed by application of mouse APAAP complex at a dilution of $1 / 1(\mathrm{vol} / \mathrm{vol})$ for 30 minutes. After washing in TBS, the last two steps were repeated for 10 minutes each. The colour was developed by 20 minutes of incubation with new Fuchsin solution.

\section{Microvessel and lymphatic vessel counting}

Microvessel and lymph vessel counting was performed at the tumour invading front and in the inner tumour areas. The areas of highest vascularisation were chosen at low power $(\times 100)$ and vessel counting followed on three chosen high power $(\times 200)$ fields of the highest density. The microvessel density was the median of the vessel counts obtained in these three fields. Vessels with a clearly defined lumen or well defined linear vessel shape, but not single endothelial cells, were taken into account for microvessel counting.

\section{MIB 1 immunostaining}

In eight selected endometrial carcinoma tissue samples (considered to have the highest LYVE-1 vessel reactivity), simple LYVE-1 and double LYVE-1/MIBl staining was performed. The LYVE-1 staining procedure was performed as described above, whereas for double staining the MIB-1 antibody (clone PRO224; YLEM, Rome, Italy) was applied immediately before the colour development, and incubated overnight. After washing with TBS, sections were incubated with a secondary rabbit antimouse antibody (Kwik Biotinylated Secondary; Shandon-Upshaw) for 15 minutes and washed in TBS. Kwik streptavidin peroxidase reagent (Shandon-Upshaw) was applied for 15 minutes and the sections were again washed in TBS. The colour was developed by a 15 minute incubation with DAB solution and sections were weakly counterstained with haematoxylin. Because LYVE-1 does not stain nuclei, such a double staining, in direct comparison with parallel sequential sections stained with LYVEl alone, allowed the detection of proliferating lymphatic endothelia.

The number of lymphatic vessels with and without nuclear reactivity was recorded in three $\times 200$ optical fields in the invading tumour front and in three areas within the myometrium (away from the invading tumour area). The mean value was calculated.

\section{Statistical analysis}

Statistical analysis was performed using GraphPad Prism ${ }^{\circledR}$ 2.01 software (San Diego, California, USA). The unpaired two tailed $t$ test was used for testing relations between categorical tumour variables (vascular and lymphatic densities compared as continuous variables). All $\mathrm{p}$ values are two sided and $\mathrm{p}$ values $<0.05$ were considered to be significant.

\section{RESULTS}

\section{LYVE- 1 staining in normal tissues}

The expression of LYVE-1 was examined in normal small intestine (used as staining control), and also in the normal uterus and the normal lung. Clear staining of submucosal lymphatics, but not of the adjacent blood vessels, was noted in the intestine. This exclusive expression of LYVE-1 in the lymphatics was also confirmed in the bronchial submucosa and the myometrium. In contrast, there were no LYVE-1 positive vessels in normal endometrial and alveolar tissues, whereas a dense CD31 positive vascular network was noted in both these tissues.

\section{LYVE- 1 vessel staining}

In malignant endometrium, the mean microvessel density for each $\times 200$ optical field (using the anti-CD31 MoAb) was 29 (range, 4-77) in the tumour invading front, and this was dramatically reduced in inner tumour areas (median, 11; range, $1-42$ ). The mean microvessel density in lung carcinomas (assessed by anti-CD31 staining) was 29 (range, 6-64) in the invading front and 15 (range, 3-50) in inner tumour areas.

The tumorous vessels within the tumour body of endometrial and lung carcinomas were completely negative for LYVE-1. Myometrial lymphatics were clearly noted at the invading tumour front of some tumours (fig lA), whereas in others the lymphatics were seen at a distance of about one $\times 200$ optical field from the invading tumour front, suggestive of exclusion or even destruction of the lymph vessels by the invading tumour. Lymph vessel density at the invading tumour front ranged from 0 to 7 vessels (median, 2) for each $\times 200$ optical field. In the myometrium adjacent to the tumour invading zone, the lymph vessel density ranged from 10 to 35 (median, 22), whereas in myometrial areas away from the tumour front it ranged from 32 to 41 (median, 35) $(\mathrm{p}<0.0001)$, apparently reflecting variation in the normal regional distribution. In lung cancer, where alveolar tissue was deprived of lymphatics, LYVE-1 positive vessels were only noted in areas adjacent to entrapped bronchi. LYVE-1 positive areas, with a rather degenerating morphology, were occasionally noted among cancerous glandular structures or nests, and may correspond to incorporated lymphatic structures undergoing regression and breakdown.

\section{Lymphatic proliferation}

In eight cases of endometrial carcinoma, where the presence of lymphatics at the invading tumour front was confirmed, 


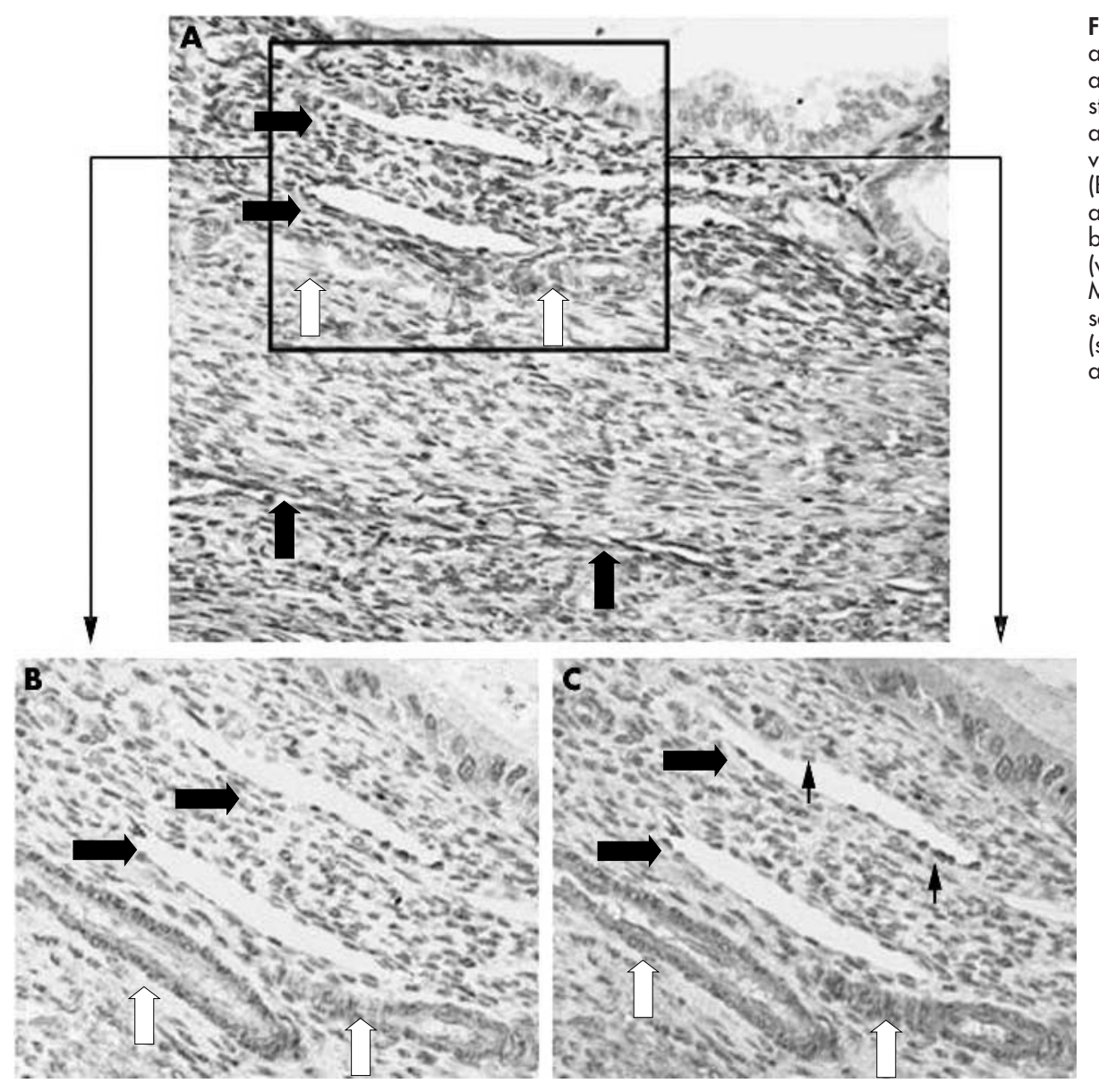

Figure 1 (A) LYVE-1 immunostaining at the invading front of an endometrial adenocarcinoma. Note the positive staining of lymphatics (large black arrows), whereas adjacent blood vessels are negative (white arrows). (B) CD31 immunostaining of the same area exhibiting blood vessel (large black arrows) but not lymphatic vessel (white arrows) reactivity. (C) LYVE-1/ $\mathrm{MIB} 1$ double immunostaining of the same area. Note MIB-1 stained nuclei (small black arrows) of both lymphatic and vascular endothelium.

double staining with MIBl and LYVE-1 revealed that lymphatic endothelial cells were actively proliferating. In the normal myometrium, the median lymphatic vessel density was 36 (range, 31-40), and the median lymphatic vessel density with MIBl nuclear staining was 0 (range, 0-2), giving a median percentage of $0 \%$ ( 0 of 36 lymphatics; $\mathrm{p}<0.0001)$. In the myometrium proximal to the tumour edge, the median lymphatic vessel density was 23 (range, 1335 ) and the median MIBl positive lymphatic vessel density was 2 (range, 1-4), which gives a median percentage of $9 \%$ (two of 23 lymphatics). At the invading tumour edge, the median lymphatic density was 2 (range, 0-6), and was accompanied by intense proliferation of lymphatics (MIBl nuclear staining: range, 0-4; median, 1; median percentage, 14\%). Figure 1A shows LYVE-1 positive lymphatics and LYVE-1 negative blood vessels in the invading tumour area, whereas fig $1 \mathrm{~B}$ shows MIB-1 nuclear staining of the vascular and lymphatic endothelium.

\section{DISCUSSION}

The production of angiogenic factors by tumour and stromal cells leads to endothelial cell migration, endothelial cell proliferation, and the formation of new tube-like structures, which sprout from preexisting capillaries of the host tissue adjacent to the invading tumour front. Newly formed and parental blood vessels are subsequently incorporated into the growing tumour mass, forming a unique vascular network composed of mature and immature vessels, which have an irregular distribution, discontinuities, shunts, and collapsed ill functioning branches. Arterial branches and disrupted veins communicate with this immature vascular network, and thereafter drain into the veins of the systemic circulation.
In contrast to the process of haemangiogenesis, less information is at present available with regard to lymphangiogenesis. The growth factor VEGF-C, produced by several different tumours, ${ }^{3}$ was thought to induce proliferation of lymph vessels after binding to the VEGFR-3 receptor. However, because KDR, the receptor that binds to VEGF-A, VEGF-B, and VEGF-C, is also expressed by the lymphatic endothelium, ${ }^{16}$ these VEGFs could be lymphangiogenic also. The formation of a new lymphatic network within tumours would greatly facilitate tumour growth by draining waste products of metabolism and promoting lymphatic dissemination of tumour cells.

\section{"A striking absence of LYVE-1 positive lymphatics was noted within the tumour body of all cases studied, even though a CD31 positive vascular network was consistently noted"}

Using the lymph vessel specific LYVE-1 MoAb, which recognises an endocytic receptor for hyaluronan, ${ }^{8}$ we assessed whether lymphangiogenesis does indeed occur in certain human malignancies, namely endometrioid adenocarcinoma of the uterus, lung adenocarcinoma, and lung squamous cell carcinoma. Staining of normal lung, myometrium, and small intestine confirmed the specificity of LYVE-1 immunostaining of lymphatics. LYVE-1 positive lymphatics were also identified within the normal myometrium and the normal bronchial submucosa. LYVE-1 positive lymphatic vessels were not seen in the normal alveolar tissue or within the normal endometrium. These findings demonstrate the variable nature of the lymphatic network among different tissues. 
A striking absence of LYVE-1 positive lymphatics was noted within the tumour body of all cases studied, even though a CD31 positive vascular network was consistently noted. In the invading edge of endometrial cancer two different patterns could be identified, namely: (1) LYVE-1 positive vessels entrapped by the spreading tumour, and (2) absence of LYVE-l positive vessels. In lung cancer, such a feature was noted only at invading tumour edges adjacent to bronchi and not adjacent to alveolar tissue, where lymphatics were absent. The lack of immunohistochemically identifiable lymphatics within the main tumour mass suggests that even though lymphatics may be incorporated into the tumour mass, these cannot survive, but rather regress and vanish. Indeed, we showed previously that the survival of vessels in the inner tumour areas is problematic, and that the vessel density rapidly decreases in tumour areas $4-6 \mathrm{~mm}$ distant from the invading tumour front. ${ }^{17}$ Cancer sections stained for lymphatics did occasionally show LYVE-1 positive stromal areas, squeezed among malignant tissue, that may represent degenerative lymphatic structures incorporated into the tumour.

Assessment of the proliferation status of endothelial cells at the invading front of this type of endometrial carcinoma exhibiting a growth pattern with incorporating lymphatics showed that lymphatic endothelium (similar to non-lymphatic vascular endothelium) had a higher proliferation rate than the endothelium located at distal areas. This shows that lymphangiogenesis may occur in the invading tumour edge, where tumour interacts with the lymphatic rich normal tissue. However, this was not a constant feature and it may differ among tumours. The lack of intratumorous lymphangiogenesis in human melanomas suggested in a study by Fallowfield and Cook, where double staining for UEAl and collagen IV was used to discriminate between blood and lymphatic vessels, agrees in part with our findings. ${ }^{18}$ In contrast, two recent studies in melanoma (using the antiLYVE-1 MoAb) confirmed the presence of focal areas exhibiting intratumorous lymphatic proliferation. ${ }^{19} 20$ Importantly, increased lymphatic density was linked with tumours with a lower proliferative activity and was associated with a better prognosis, ${ }^{20}$ showing that more aggressive tumours destroy the lymphatic vessels once incorporated, instead of stimulating their proliferation. A recent study in breast cancer showed that there are no lymphatics within the tumours, and that there was no lymphatic proliferation even in areas of intense haemangiogenesis. ${ }^{21}$ These findings are in accordance with our present study, where lymphangiogenesis or the integration of viable normal lymphatics into the tumorous vascular network did not occur within the tumour body. Careful evaluation of the invading tumour edge, however, revealed that some tumours do incorporate proliferating lymphatics, exhibiting a lymphatic network exclusively located in this thin layer (4-6 mm) of normal-tumorous interacting tissue. However, in some tumours, such as squamous cell head and neck carcinomas, lymphangiogenesis does occur within the tumour body. ${ }^{22}$ It seems that the patterns of lymphangiogenesis vary among malignancies.

The lack of both peripheral lymphangiogenesis and of an incorporated viable lymphatic network within the tumour body in most endometrial and lung tumours raises questions about how lymphatic metastasis is achieved and how lymph produced by malignant tissues is drained. One possibility regarding fluid drainage is that the lymphatics are bypassed and that the host's venous supply carries out the process. In that way, tumours may use their vasculature both in terms of a venous and a lymphatic network. Alternatively, the lymph produced by peripheral tumour areas could be drained into more distal host lymphatics in the surrounding normal
Take home messages

- Using specific immunostaining with the anti-LYVE-1 monoclonal antibody and the panendothelial marker CD31 in malignant pulmonary and endometrial tissues, we found that although lymphangiogenesis can occur at the invading tumour front, incorporated lymphatics do not survive

- Therefore, the dissemination of cancer cells through the lymphatics may occur by invasion of peripheral cancer cells into the adjacent normal lymphatics, or through shunts eventually produced at the invading tumour front as a consequence of active angiogenesis and lymphangiogenesis

tissue. Therefore, lymphatic dissemination of cancer may occur by active invasion of cancer cells from the invading tumour front into the host's lymphatics, as Hartveit et al suggested in $1990 .{ }^{23}$ In cases with active lymphangiogenesis at the invading edge, such invasion would be more probable, and shunts among the sprouting lymphatic and nonlymphatic endothelium may also occur. In this way, invasion of cancer cells in non-lymphatic vessels could also result in node metastasis. Whether peripheral lymphangiogenesis is necessary or enhances the probability of lymphatic metastasis cannot be concluded from our present study because the endometrial carcinomas studied were stage I.

In conclusion, using specific immunostaining for LYVE-1 and the proliferation marker MIB1, lymphangiogenesis was detected in a subset of endometrial adenocarcinomas, and was located exclusively at the invading tumour edge. Such an event is difficult to confirm in lung carcinomas because alveolar tissue is deprived of vessels and such a process should be sought only in the proximity of tumour to bronchial structures. The lack of lymphatics within the tumour body, regardless of the presence or absence of peripheral lymphangiogenesis, suggests that tumour lymph drains directly into the immature intratumorous vascular network, and only in peripheral tumour areas is the lymph drained into the lymphatics of the surrounding normal tissue. Lymphatic dissemination of cancer cells may occur either by active invasion of cancer cells into host lymphatics adjacent to tumour tissue or by invasion of actively dividing and intercommunicating non-lymphatic and lymphatic vessels generated at the invading tumour edge.

\section{Authors' affiliations}

M I Koukourakis, A Giatromanolaki, E Sivridis, C Simopoulos, Tumour and Angiogenesis Research Group, Departments of Radiotherapy/ Oncology, Pathology, and Surgery, Democritus University of Thrace, Medical School, Alexandroupolis 68100, Greece

K C Gatter, Department of Pathology, Nuffield Department of Clinical Laboratory Sciences, John Radcliffe Hospital, Oxford OX3 9DS, UK A L Harris, Cancer Research UK, Molecular Oncology Laboratories, Weatheral Institute of Molecular Medicine, John Radcliffe Hospital D G Jackson, MRC Human Immunology Unit, Institute of Molecular Medicine, Oxford Radcliffe Hospital, Headington, Oxford OX3 9DS, UK

\section{REFERENCES}

1 Vermeulen PB, Gasparini G, Fox SB, et al. Quantification of angiogenesis in solid tumors: an international consensus on the methodology and criteria of evaluation. Eur J Cancer 1996;14:2474-84.

2 Parums DV, Cordell JL, Micklem K, et al. JC70: a new monoclonal antibody that detects vascular endothelium associated antigen on routinely processed tissue sections. J Clin Pathol 1990:43:752-7.

3 Paavonen K, Puolakkainen P, Jussila L, et al. Vascular endothelial growth factor receptor-3 in lymphangiogenesis in wound healing. Am J Pathol 2000; 156:1499-504. 
4 Olofsson B, Jeltsch M, Eriksson U, et al. Current biology of VEGF-B and VEGFC. Curr Opin Biotechnol 1999;10:528-35.

5 Kaipainen A, Korhonen J, Mustonen T, et al. Expression of the fms-like tyrosine kinase 4 gene becomes restricted to lymphatic endothelium during development. Proc Natl Acad Sci U S A 1995:92:3566-70.

6 Lymboussaki A, Olofsson B, Eriksson U, et al. Vascular endothelial growth factor (VEGF) and VEGF-C show overlapping binding sites in embryonic endothelia and distinct sites in differentiated adult endothelia. Circ Res 1999;85:992-9.

7 Partanen TA, Alitalo K, Miettinen M. Lack of lymphatic vascular specificity of vascular endothelial growth factor receptor 3 in 185 vascular tumors. Cancer 1999;86:2406-12.

8 Banerii S, Ni J, Wang SX, et al. LYVE-1, a new homologue of the CD44 glycoprotein, is a lymph-specific receptor for hyaluronan. J Cell Biol 1999; 144:789-801.

9 Prevo R, Banerii S, Ferguson D, et al. Mouse LYVE-1 is an endocytic receptor for hyaluronan in lymphatic endothelium. J Biol Chem 2001;276:19420-30.

10 Fraser JR, Kimpton WG, Laurent TC, et al. Uptake and degradation of hyaluronan in lymphatic tissue. Biochem $J$ 1998;256:153-8.

11 Laurent TC, Fraser JR. Hyaluronan. FASEB J 1992;6:2397-404.

12 Clarijs R, Ruiter DJ, de Waal RM. Lymphangiogenesis in malignant tumours: does it occur? J Pathol 2001;193:143-6.

13 Pepper MS. Lymphangiogenesis and tumor metastasis: myth or reality? Clin Cancer Res 2001;7:462-8.

14 Karpanen T, Egeblad M, Karkkainen MJ, et al. Vascular endothelial growth factor $C$ promotes tumor lymphangiogenesis and intralymphatic tumor growth. Cancer Res 2001;61:1786-90.
15 Skobe M, Hawighorst T, Jackson DG, et al. Induction of tumor lymphangiogenesis by VEGF-C promotes breast cancer metastasis. Nat Med 2001;7:192-8.

16 Skobe $M$, Brown LF, Tognazzi $\mathrm{K}$, et al. Vascular endothelial growth factor-C (VEGF-C) and its receptors KDR and flt- 4 are expressed in AIDS-associated Kaposi's sarcoma. Invest Dermatol 1999;113:1047-53.

17 Giatromanolaki A, Koukourakis MI, Sivridis E, et al. Invading edge vs. inner (edvin) patterns of vascularisation: an interplay between angiogenic and vascular survival factors defines clinical behaviour of non small cell lung cancer. J Pathol 2000;192:140-9.

18 Fallowfield ME, Cook MG. Lymphatics in primary cutaneous melanoma. Am J Surg Pathol 1990;14:370-4.

19 Dadras SS, Paul T, Bertoncini J, et al. Tumor lymphangiogenesis: a novel prognostic indicator for cutaneous melanomametastasis and survival. Am J Pathol 2003;162:1951-60.

20 Straume O, Jackson DG, Akslen LA. Independent prognostic impact of lymphatic vessel density and presence of low-grade lymphangiogenesis in cutaneous melanoma. Clin Cancer Res 2003;9:250-6.

21 Williams CS, Leek RD, Robson AM, et al. Absence of lymphangiogenesis and intratumoural lymph vessels in human metastatic breast cancer. J Pathol 2003;200:195-206.

22 Beasley NJ, Prevo R, Banerji S, et al. Intratumoral lymphangiogenesis and lymph node metastasis in head and neck cancer. Cancer Res 2002;62:1315-20.

23 Hartveit $F$. Attenuated cells in breast stroma: the missing lymphatic system of the breast. Histopathology 1990; 16:533-43. 